\title{
Gravity-Based Characterization of Three-Axis Accelerometers in Terms of Intrinsic Accelerometer Parameters
}

\author{
Jon Geist ${ }^{1}$, Muhammad Yaqub Afridi ${ }^{1}$, Craig D. McGray ${ }^{1,2}$, and Michael Gaitan ${ }^{1}$ \\ ${ }^{1}$ National Institute of Standards and Technology, \\ Gaithersburg, MD 20899, USA \\ ${ }^{2}$ Modern Microsystems Inc., \\ Silver Spring, MD 20904, USA \\ jon.geist@nist.gov \\ afridi@nist.gov \\ craig@modernmicrosystems.com \\ michael.gaitan@nist.gov
}

\begin{abstract}
Cross-sensitivity matrices are used to translate the response of three-axis accelerometers into components of acceleration along the axes of a specified coordinate system. For inertial three-axis accelerometers, this coordinate system is often defined by the axes of a gimbal-based instrument that exposes the device to different acceleration inputs as the gimbal is rotated in the local gravitational field. Therefore, the cross-sensitivity matrix for a given three-axis accelerometer is not unique. Instead, it depends upon the orientation of the device when mounted on the gimbal. We define nine intrinsic parameters of three-axis accelerometers and describe how to measure them directly and how to calculate them from independently determined cross-sensitivity matrices. We propose that comparisons of the intrinsic parameters of three axis accelerometers that were calculated from independently determined cross-sensitivity matrices can be useful for comparisons of the cross-sensitivity-matrix measurement capability of different institutions because the intrinsic parameters will separate the accelerator-gimbal alignment differences among the participating institutions from the purely gimbal-related differences, such as gimbal-axis orthogonality errors, $z$-axis gravitational-field alignment errors, and angle-setting or angle-measurement errors.
\end{abstract}

Key words: accelerometer; calibration; characterization; gimbal; gravity; three-axis.

Accepted: June 14, 2017

Published: July 13, 2017; Current Version: March 6, 2019

https://doi.org/10.6028/jres.122.032

\section{Introduction}

There are many ways to carry out measurements of the inertial response of three-axis accelerometers [1-5]. The most accurate methods rely on selecting optimal values for sets of adjustable parameters in over-determined models of accelerometer response to measurements taken with different orientations of the device under test (DUT) ${ }^{1}$ relative to the gravitational field or some other accurately defined acceleration. The purpose of this manuscript is to describe a new gimbal-based measurement and analysis method employing an over-determined model of the response as a function of orientation of each of the accelerometers in the local gravitational field.

\footnotetext{
${ }^{1} \mathrm{~A}$ three-axis accelerometer will be referred to as a device under test (DUT) in this article, reserving the word "accelerometer" to refer to each of the three individual accelerometers that comprise the DUT.
} 
Both the measurement method and the analysis method are optimized for estimating the intrinsic parameters (defined below) of a DUT. These parameters are expected to be useful for interpreting the results of multiple-laboratory comparisons of three-axis-accelerometer calibration capability and are complementary to the cross-sensitivity matrix method widely used in applications of three-axis accelerometers. Understanding the relation of our work to the work cited in the references presupposes knowledge of our results, so it is postponed to the discussion at the end of the main text.

We define three coordinate systems as follows: a world-referenced coordinate system, $(\hat{X}, \hat{Y}, \hat{Z})$, a gimbal-referenced coordinate system, $(\hat{x}, \hat{y}, \hat{z})$, and a device-referenced coordinate system, $(\hat{u}, \hat{v}, \hat{w})$. Since the analysis presented here is independent of the origins of these coordinate systems, we choose the origins to coincide at a point at the center of the top of the DUT, as illustrated in Fig. 1.

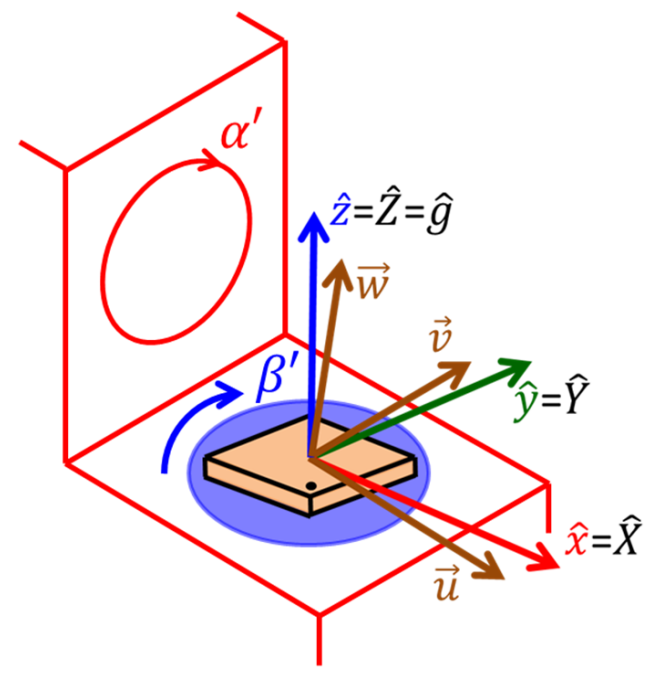

Fig. 1. Diagram of a gimbal composed of a $\beta$-rotation stage (blue ellipse) located on an $\alpha$-rotation stage (red outline). A DUT (tan box) composed of three nominally orthogonal accelerometers is mounted on the $\beta$ stage. Unit vectors $\hat{x}, \hat{y}$, and $\hat{z}$, which are parallel to the corresponding orthogonal $X, Y$, and $Z$ axes of the world coordinate system, are shown in red, green, and blue, respectively. The brown arrows represent vectors $\vec{u}, \vec{v}$, and $\vec{w}$, which encode the magnitude and direction of maximum responsivity of the respective $u-, v-$, and $w$-axis accelerometers. It is assumed that the accelerometer and gimbal axes are approximately aligned as shown in the figure.

The positive directions of the rotation angles $\alpha^{\prime}$ and $\beta^{\prime}$ of the $\alpha$ and $\beta$ stages are shown in Fig. 1 in the appropriate color. Angles with primes are used to describe the rotation of the gimbal and DUT relative to the gravitational field. Angles without primes are used to describe the rotation of the gravitational field relative to the DUT and gimbal. The vectors $\vec{u}, \vec{v}$, and $\vec{w}$ point in the directions of maximum responsivity of the $u-, v-$, and $w$-axis accelerometers. The magnitude of each of these vectors is the intrinsic responsivity of the corresponding accelerometer. The $(X, Y, Z)$ coordinate system is an orthonormal coordinate system that is fixed relative to the surface on which the gimbal is mounted. The $Z$ axis of this system is aligned with the local gravitational field. The $(x, y, z)$ coordinate system is an orthonormal system that is fixed with respect to the $\beta$ stage of the gimbal, and therefore fixed with respect to the DUT. This system is defined to be coincident with the $(X, Y, Z)$ coordinate system when the gimbal is in the orientation shown. This orientation will be referred to as configuration 1.

The derivations in this manuscript are based on three important assumptions that will be introduced and repeated as needed. To start, assume that there is a different unique axis along which each of the 
three DUT accelerometers has a maximum response to the gravitational field, and represent these unique axes by $u, v$, and $w$. With this assumption, three sets of intrinsic parameters of the DUT can be defined, each consisting of three intrinsic parameters. The first set consists of the angles between the $u, v$, and $w$ axes, which in general will not be perfectly orthogonal. The second set of intrinsic parameters consists of the magnitudes of the responsivities of the $u$-, $v$-, and $w$-axis accelerometers along the $u, v$, and $w$ axes, respectively. The third set consists of the offset signals of the respective accelerometers in the absence of acceleration input stimuli.

Table 1. Key concepts, parameters, and conventions used in this manuscript.

\begin{tabular}{|c|c|c|}
\hline \multicolumn{3}{|c|}{ Definitions and Conventions used in this Manuscript } \\
\hline Symbol & Definition & Context \\
\hline$\overline{\hat{x}, \hat{y}, \hat{z}}$ & Unit vectors in gimbal coordinate system & See Figs. 1 and 2 \\
\hline$\alpha_{x}^{\prime}, \alpha_{y}^{\prime}, \beta_{z}^{\prime}$ & Angles of rotation around gimbal axes & See Figs. 1 and 2 \\
\hline$-\alpha_{x},-\alpha_{y},-\beta_{z}$ & Same angles, but from DUT point of view & See Figs. 1 and 2 \\
\hline$\hat{u}, \hat{v}, \hat{w}$ & Unit vectors in DUT coordinate system & See Figs. 1 and 2 \\
\hline$\vec{u}, \vec{v}, \vec{w}$ & Intrinsic responsivity vectors & See Figs. 1 and 2 \\
\hline$\phi_{u v}, \phi_{v w}, \phi_{w u}$ & Intrinsic angles between DUT unit vectors $\hat{u}, \hat{v}, \hat{w}$ & $\hat{u} \cdot \hat{v}=\cos \left(\phi_{u v}\right)$ \\
\hline$r$ & Meta-symbol that represents either $u, v$, or $w$ & $\vec{r}=r \hat{r}=r_{x} \hat{x}+r_{y} \hat{y}+r_{z} \hat{z}$ \\
\hline$s$ & Meta-symbol that represents either $x, y$, or $z$ & $r_{s}, \hat{s}, \theta_{s}, \rho_{s s^{\prime}}$ \\
\hline$O_{r}$ & Accelerometer $r$ output with 0 acceleration input & Output offset signal \\
\hline$\vec{a}$ & General acceleration input experienced by DUT & $\vec{a}=a_{x} \hat{x}+a_{y} \hat{y}+a_{z} \hat{z}$ \\
\hline$\theta_{s}$ & Meta-symbol that represents either $\alpha_{x}, \alpha_{y}$, or $\beta_{z}$ & $R_{r}\left(\theta_{S}\right)$ \\
\hline$n=1, \ldots, N$ & Index of number of rotation angles & $\alpha_{x n}, \alpha_{y n}, \beta_{z n}$ \\
\hline & Acceleration due to gravity & DUT input acceleration \\
\hline$\vec{g}\left(\theta_{s n}\right)$ & Gravity vector as experienced by DUT & as function of $\theta_{s n}$ \\
\hline$m=1, \ldots, M$ & Index of the number of response measurements & at each angle \\
\hline$\varepsilon_{r s n m}$ & Noise in $m^{\text {th }}$ output value of accelerometer $r$ & at angle $\theta_{s n}$ \\
\hline$R_{r m}\left(\theta_{s n}\right)$ & $\begin{array}{l}m^{t h} \text { value }(m=1, \cdots, M) \text { of the measured DUT } \\
\text { response that contributes to average value }\end{array}$ & $R_{r}\left(\theta_{s n}\right)=(1 / M) \sum_{1}^{M} R_{r m}\left(\theta_{s n}\right)$ \\
\hline$R_{r}\left(\theta_{s n}\right)$ & Average response of DUT accelerometer $r$ & as function of $\theta_{s n}$ \\
\hline$S E\left(R_{r}\right)$ & Standard-error uncertainty of mean & $R_{r} \pm K S E\left(R_{r}\right)$ \\
\hline$K$ & Coverage factor of uncertainty expression & $K=2$ \\
\hline $\mathscr{P}$ & Cross-sensitivity matrix in gimbal coordinates & $\mathscr{P}=\left[\begin{array}{lll}\rho_{x x} & \rho_{x y} & \rho_{x z} \\
\rho_{y x} & \rho_{y y} & \rho_{y z} \\
\rho_{z x} & \rho_{z y} & \rho_{z z}\end{array}\right]$ \\
\hline $\mathscr{Q}$ & Inverse cross-sensitivity matrix & $\mathscr{Q}=\mathscr{P}^{-1}=\left[\begin{array}{lll}q_{x x} & q_{x y} & q_{x z} \\
q_{y x} & q_{y y} & q_{y z}\end{array}\right]$ \\
\hline$[\vec{r} \cdot \hat{s}]=\mathscr{Q}$ & Cross-responsivity matrix in mixed coordinates & {$[\vec{r} \cdot \hat{s}]=\left[\begin{array}{ccc}u_{x} & u_{y} & u_{z} \\
v_{x} & v_{y} & v_{z} \\
w_{x} & w_{y} & w_{z}\end{array}\right]$} \\
\hline
\end{tabular}

Table 1 introduces and defines the key concepts, parameters, and conventions used in this manuscript. The meta-symbol $r$ and the short-hand notation $r \in\{u, v, w\}$ will be used in a single equation or sentence to represent the $u, v$, and $w$ accelerometer or direction in the set of equations or sentences that is obtained by first replacing $r$ with $u$, then with $v$, and then with $w$ in the same single equation or sentence. 
The same convention will be used for the meta-symbols $s \in\{x, y, z\}$ and $\theta_{s}^{\prime} \in\left\{\alpha_{x}^{\prime}, \alpha_{y}^{\prime}, \beta_{z}^{\prime}\right\}$, where $\alpha_{x}^{\prime}, \alpha_{y}^{\prime}$, and $\beta_{z}^{\prime}$ are the angle of rotation around axes parallel to the $x, y$, and $z$ axes of the gimbal, respectively. These rotation angles as seen from the point of view of the DUT are given by $\theta_{s}=-\theta_{s}^{\prime}$.

Now assume that the maximum responsivity of each accelerometer (zero-offset-corrected signal per unit input acceleration when aligned with the gravitational field) and the direction of maximum responsivity of each accelerometer are both independent of acceleration. With this assumption of linearity, the responsivity of the $r \in\{u, v, w\}$ accelerometer can be expressed as a vector

$$
\vec{r}=r \hat{r}=r_{x} \hat{x}+r_{y} \hat{y}+r_{z} \hat{z}
$$

in the $(u, v, w)$ coordinate system, as in the middle expression in Eq. (1), or it can be expressed as a vector in the $(x, y, z)$ coordinate system as in the right-most expression in this equation, where $r=\|\vec{r}\|$ is the magnitude of the vector $\vec{r}$, and the unit vector $\hat{r}=\vec{r} / r$ points in the direction of maximum responsivity. Thus, $\vec{r}$, its magnitude, its direction, and its elements in the $(u, v, w)$ coordinate system ${ }^{2}$ are intrinsic parameters of the DUT. This idea will be developed further in what follows.

Next, we define the response vector $\vec{R}_{r}$ of the $r$-axis accelerometer as

$$
\vec{R}_{r}=R_{r x} \hat{x}+R_{r y} \hat{y}+R_{r z} \hat{z} .
$$

This vector, which describes the response along the direction of maximum response of the $r$-axis accelerometer, is useful here because it can be expressed in the $(x, y, z)$ coordinate system of the gimbal, as can the acceleration vector

$$
\vec{a}=a_{x} \hat{x}+a_{y} \hat{y}+a_{z} \hat{z}
$$

However, $R_{r}$ is not useful in general because $\vec{R}_{r} \neq R_{r} \hat{r}$ in general. Only in the special cases where $a_{x}=a_{y}=a_{z}$ or $\hat{u}=\hat{x}, \hat{v}=\hat{y}$, and $\hat{w}=\hat{z}$ does the equality in Eq. (2) hold.

Next, assume that the errors associated with (1) the alignment of the gimbal $z$-axis with the local gravitational field, (2) the orthogonality of the gimbal axes, and (3) the gimbal angle measurements are all negligible. Based on this and the prior assumptions, it is useful to fit a linear model of the response of each accelerometer to the measured response as a function of gimbal-rotation angle. We define $\alpha^{\prime}$ to be the rotation angle of the outer gimbal axis and $\beta^{\prime}$ to be the rotation angle of the inner gimbal axis. Depending on the initial configuration of the DUT, $\alpha^{\prime}$ may be considered to be either the $x$-axis of the gimbal or the $y$-axis of the gimbal. When $\alpha^{\prime}$ represents the $x$-axis, we write $\alpha_{x}^{\prime}=\alpha^{\prime}$. Alternatively, when $\alpha^{\prime}$ represents the $y$-axis, we write $\alpha_{y}^{\prime}=\alpha^{\prime}$. The inner axis of the gimbal is always taken to be the $z$-axis, so we write $\beta_{z}^{\prime}=\beta^{\prime}$. Figure 2 shows the three starting configurations of the DUT for the response measurements.

With sufficient replication of the individual measurements of response at each set of angles, the difference between the standard error of the fit and the standard error of the individual measurements at each angle will determine the extent to which the above assumptions have been satisfied. This idea and various sources of error not included in the model are discussed in more detail in Appendix B. The remainder of this report comprises six sections, "Measurement Methods," "Analysis Methods," "Relation of the intrinsic parameters to the conventional cross-sensitivity matrix," "Relation to Previous Work," "Discussion and Conclusion," and two appendices.

\section{Measurement Method}

The measurement method consists of recording the response of each axis accelerometer and the rotation angle as the DUT is separately rotated through $360^{\circ}$ around each of the three orthogonal axes

${ }^{2}$ This does not apply to its elements in either the world coordinate system or the gimbal coordinate system. 
of the gimbal $\alpha_{x}^{\prime}, \alpha_{y}^{\prime}$, and $\beta_{z}^{\prime}$. The measurement will be described in terms of the DUT-gimbal configuration shown in Fig. 1. This configuration will be referred to as configuration 1, and all measurements begin from this configuration.

Red, green, and blue arrows represent orthogonal unit vectors $\hat{x}, \hat{y}$, and $\hat{z}$, respectively, in a gimbal-based coordinate system, which is defined to coincide with the unit vectors $(\hat{X}, \hat{Y}, \hat{Z})$ of the stationary, orthogonal coordinate system when the DUT is in orientation A of Fig. 2.

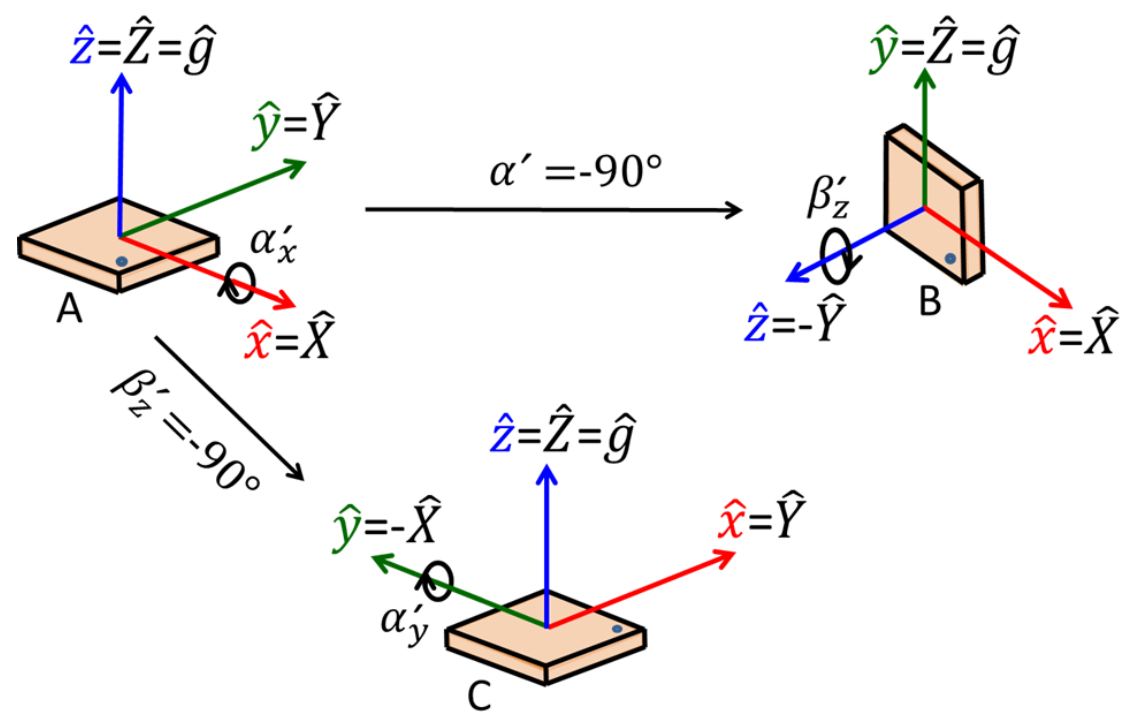

Fig. 2. A, B, and C: Starting DUT orientations for measurements of accelerometer response during rotations $\alpha_{x}^{\prime}, \alpha_{y}^{\prime}$, and $\beta_{z}^{\prime}$ around gimbal axes $x, y$, and $z$, respectively.

Orientation A in Fig. 2 is the DUT orientation obtained with configuration 1 of Fig. 1. The rotations around gimbal axes $\hat{x}$ and $\hat{z}$ required to obtain starting orientations $\mathbf{B}$ and $\mathbf{C}$ from starting orientation $\mathbf{A}$ are also shown in Fig. 2. It is assumed that the gimbal unit vector $\hat{z}$ is perfectly aligned with the gravitational unit vector $\hat{g}=\vec{g} /\|g\|$ in $\mathbf{A}$ and $\mathbf{C}$, whereas it is the gimbal unit vector $\hat{y}$ that is perfectly aligned with $\hat{g}$ in $\mathbf{B}$. Note that all three coordinate systems are right handed, but that $\alpha_{x}^{\prime}$ and $\beta_{z}^{\prime}$ follow left-hand rotation rules, whereas $\alpha_{y}^{\prime}$ follows a right-hand rule. This choice was a compromise to retain the angle measuring conventions of the gimbal and obtain the same form for the three equations given as Eq. (5) below.

If the $\vec{u}, \vec{v}$, and $\vec{w}$ vectors are not orthogonal to each other, then they cannot, even in principle, be perfectly aligned with the respective orthonormal $\hat{x}-, \hat{y}$-, and $\hat{z}$-unit vectors of the gimbal. However, as shown in Eq. (1), they can always be expressed in terms of the gimbal coordinate system as

$$
\begin{aligned}
& \vec{u}=u_{x} \hat{x}+u_{y} \hat{y}+u_{z} \hat{z}, \text { where } u=\|\vec{u}\|=\sqrt{u_{x}^{2}+u_{y}^{2}+u_{z}^{2}} \text { is the magnitude of } \vec{u}, \\
& \vec{v}=v_{x} \hat{x}+v_{y} \hat{y}+v_{z} \hat{z}, \text { where } v=\|\vec{v}\|=\sqrt{v_{x}^{2}+v_{y}^{2}+v_{z}^{2}} \text { is the magnitude of } \vec{v}, \\
& \vec{w}=w_{x} \hat{x}+w_{y} \hat{y}+w_{z} \hat{z}, \text { where } w=\|\vec{w}\|=\sqrt{w_{x}^{2}+w_{y}^{2}+w_{z}^{2}} \text { is the magnitude of } \vec{w} .
\end{aligned}
$$

Next assume that the $z$ axis of the gimbal has been aligned with gravity ${ }^{3}$ to the desired uncertainty

\footnotetext{
${ }^{3}$ We adopt the widely used convention that the gravitational acceleration vector points up, which is opposite to the direction of the gravitational force vector.
} 


\section{Journal of Research of National Institute of Standards and Technology}

as described in detail in [6] or Appendix A.

Refer now to Fig. 2, which illustrates the measurement process. The gimbal is rotated separately through angles $\alpha_{x n}^{\prime}, \alpha_{y n}^{\prime}$, and $\beta_{z n}^{\prime}$ starting from positions $\mathbf{A}, \mathbf{C}$, and $\mathbf{B}$, respectively, for $n=1, \ldots, N$. The dependencies of the gravitational vector $\vec{g}$ on the corresponding angles $\alpha_{x n}=-\alpha_{x n}^{\prime}, \alpha_{y n}=-\alpha_{y n}^{\prime}$, and $\beta_{z n}=-\beta_{z n}^{\prime}$, which are defined from the point of view of the DUT, are given by

$$
\begin{aligned}
& \vec{g}\left(\alpha_{x n}\right)=-g \sin \left(\alpha_{x n}\right) \hat{y}+g \cos \left(\alpha_{x n}\right) \hat{z}, \\
& \vec{g}\left(\alpha_{y n}\right)=-g \sin \left(\alpha_{y n}\right) \hat{x}+g \cos \left(\alpha_{y n}\right) \hat{z}, \\
& \vec{g}\left(\beta_{z n}\right)=-g \sin \left(\beta_{z n}\right) \hat{x}+g \cos \left(\beta_{z n}\right) \hat{y},
\end{aligned}
$$

respectively, as shown Figs. $2 \mathbf{A}, 2 \mathbf{C}$, and $2 \mathbf{B}$, respectively. Note that this result is accurate only to the accuracy with which the angle settings correspond to the actual values of the angles and to the accuracy with which the pertinent gimbal axis has been aligned with the gravitation unit vector $\hat{g}$ in Figs. 2A-2C.

First, consider a single measurement by a single accelerometer at a single gimbal angle as a step to developing the full measurement model. For instance, consider the $m^{\text {th }}(m=1, \ldots, M)$ value of the response of the $w$-axis accelerometer to gravity at rotation angle $\theta_{s n}$, which, according to the assumption of linearity, is given by

$$
R_{w m}\left(\alpha_{x n}\right)=O_{w}+\vec{w} \cdot \vec{g}\left(\alpha_{x n}\right)+\varepsilon_{w x m n}+\delta_{w x n}=O_{w}-w_{y} g \sin \left(\alpha_{x n}\right)+w_{z} g \cos \left(\alpha_{x n}\right)+\varepsilon_{w x m n}+\delta_{w x n},
$$

where $O_{w}$ is the nominally constant offset signal from the $w$-axis accelerometer, $\varepsilon_{w x m n}$ is a normally distributed random variable with population mean, sample mean, and sample standard error of the mean given by $0, \varepsilon_{w x n}$, and $S E\left(\varepsilon_{w x n}\right)$, respectively, and $\delta_{w x n}$, which is assumed to be zero for now, is the error caused by approximating the actual response of the $w$ accelerometer by the simple three-parameter model of Eq. (6) with $\delta_{w x m n}=0$.

The $w$ dependence of Eq. (6) associates the equation with the $w$ accelerometer. The $x$ dependence associates the equation with orientation $\mathbf{A}$ in Fig. 2, and the $m$ dependence associates the equation with the $m^{\text {th }}$ measurement by the $w$ accelerometer of the response $R_{w m}\left(\alpha_{x n}\right)$ at the $n^{\text {th }}$ angle $\alpha_{x n}$.

Therefore, the average response at angle $\alpha_{x n}$ is given by

$$
\begin{aligned}
R_{w}\left(\alpha_{x n}\right)=\frac{1}{M} \sum_{m=1}^{M} R_{w m}\left(\alpha_{x n}\right) & =\frac{1}{M} \sum_{m=1}^{M}\left[O_{w}-w_{y} g \sin \left(\alpha_{x n}\right)+w_{z} g \cos \left(\alpha_{x n}\right)+\varepsilon_{w x m n}\right] \\
& =O_{w x}-w_{y} g \sin \left(\alpha_{x n}\right)+w_{z} g \cos \left(\alpha_{x n}\right),
\end{aligned}
$$

where

$$
O_{w x}=O_{w}+\frac{1}{N} \sum_{n=1}^{N} \frac{1}{M} \sum_{m=1}^{M} \varepsilon_{w x m n}=O_{w}+\frac{1}{N} \sum_{n=1}^{N} \varepsilon_{w x n}
$$

is the average offset (response to zero acceleration) of the $w$ accelerometer during rotation around gimbal axis $x$. Ideally, the average offset $O_{w x}$ would be independent of the gimbal axis about which the gimbal is rotated. In practice, the value of the mean offset signal $O_{w x}$ will vary from measurement set to measurement set due to offset drift, if any, and due to the residual noise that shows up in variations in the mean value of $\varepsilon_{w x m n}$, which is zero only in the limit as $M \rightarrow \infty$.

Equations (7) for $n=1, \ldots, N$ represents a system of linear equations that can be solved for $O_{w x}, w_{y}$, and $w_{z}$ by standard least-squares methods, and the corresponding standard (error) uncertainties $S E\left(O_{w x}\right), S E\left(w_{y}\right)$, and $S E\left(w_{z}\right)$ can be also be calculated by standard methods. Furthermore, the off-diagonal elements in the variance-covariance matrix should be negligible compared to the diagonal elements, because the terms in Eq. (7) are orthogonal over a uniformly sampled $360^{\circ}$ rotation. 
There are eight more independent equations like Eq. (7) that describe the response of each of three accelerometers to rotations of the DUT around each of a total of three gimbal axes. Therefore, there are nine systems of overdetermined linear equations

$$
\begin{aligned}
& R_{u}\left(\alpha_{x n}\right)=O_{u x}+u_{y 1} g \sin \left(\alpha_{x n}\right)+u_{z 1} g \cos \left(\alpha_{x n}\right), \\
& R_{v}\left(\alpha_{x n}\right)=O_{v x}+v_{y 1} g \sin \left(\alpha_{x n}\right)+v_{z 1} g \cos \left(\alpha_{x n}\right), \\
& R_{w}\left(\alpha_{x n}\right)=O_{w x}+w_{y 1} g \sin \left(\alpha_{x n}\right)+w_{z 1} g \cos \left(\alpha_{x n}\right), \\
& R_{u}\left(\alpha_{y n}\right)=O_{u y}+u_{x 1} g \sin \left(\alpha_{y n}\right)+u_{z 2} g \cos \left(\alpha_{y n}\right), \\
& R_{v}\left(\alpha_{y n}\right)=O_{v y}+v_{x 1} g \sin \left(\alpha_{y n}\right)+v_{z 2} g \cos \left(\alpha_{y n}\right), \\
& R_{w}\left(\alpha_{y n}\right)=O_{w y}+w_{x 1} g \sin \left(\alpha_{y n}\right)+w_{z 2} g \cos \left(\alpha_{y n}\right), \\
& R_{u}\left(\beta_{z n}\right)=O_{u z}+u_{x 2} g \sin \left(\beta_{z n}\right)+u_{y 2} g \cos \left(\beta_{z n}\right), \\
& R_{v}\left(\beta_{z n}\right)=O_{v z}+v_{x 2} g \sin \left(\beta_{z n}\right)+v_{y 2} g \cos \left(\beta_{z n}\right), \\
& R_{w}\left(\beta_{z n}\right)=O_{w z}+w_{x 2} g \sin \left(\beta_{z n}\right)+w_{y 2} g \cos \left(\beta_{z n}\right),
\end{aligned}
$$

for $n=1, \ldots, N>3$, which can be solved by the same standard method used for Eq. (7).

The first three equations in Eq. (9) describe the measured responses of the $u, v$, and $w$ accelerometers, respectively, as the gimbal is rotated around the $x$-axis. Similarly, the second three equations in Eq. (9) describe the measured responses of the $u, v$, and $w$ accelerometers, respectively, as the gimbal is rotated around the $y$-axis, and the third three equations in Eq. (9) describe the measured responses of the $u, v$, and $w$ accelerometers, respectively, as the gimbal is rotated around the $z$-axis.

Each line of Eq. (9) describes an independent system of equations like Eq. (7), because the experimental data for each line are different. If each system of linear equations represented by a single equation in Eq. (9) is solved independently, the solutions to the nine systems of equations provides two independent estimates for each $r_{s}$. The two estimates for these parameters can be represented concisely as $r_{s p}$, where the meta-symbol $r$ represents any member of the set $\{u, v, w\}$, the meta-symbol $s$ represents any member of the set $\{x, y, z\}$, and the meta-symbol $p$ represents any member of the set $\{1,2\}$ as shown in Eq. (9).

The asymmetric distribution of the 1's and 2's in Eq. (9) reflects the fact that $\hat{z}$ is parallel to $\hat{g}$ in two starting orientations (A and $\mathbf{C}$ ) in Fig. 2, whereas $\hat{y}$ is parallel to $\hat{g}$ in only one starting orientation (B), and $\hat{x}$ is not parallel to $\hat{g}$ in any starting orientation. The two independent estimates for the same parameter will be averaged over $p=1$ and $p=2$ at a later stage of the analysis.

It is also possible to solve the nine independent systems of linear equations represented by Eq. (9) simultaneously, but it may make interpretation of the residuals of the fit more difficult. However, if all of the equations represented by Eq. (9) are solved simultaneously, then the numerical subscripts in Eq. (9) should be deleted, which sets $r_{s 2}=r_{s 1}$ and provides the average values of $r_{s 1}$ and $r_{s 2}$ directly.

\section{Analysis Method}

Either simultaneously solve the nine systems of linear equations represented by Eq. (9) for the adjustable parameters $O_{r s}, r_{s^{\prime}}$, and $r_{s^{\prime \prime}}$, and calculate the corresponding standard (error) uncertainties $S E\left(O_{r s}\right), S E\left(r_{s^{\prime}}\right)$, and $S E\left(r_{s^{\prime \prime}}\right)$ for each combination of $r \in\{u, v, w\}$ and $s \neq s^{\prime} \neq s^{\prime \prime} \in\{x, y, z\}$,

or independently solve each system for the adjustable parameters $O_{r s}, r_{s^{\prime}}$, and $r_{s^{\prime \prime}} p^{\prime}$, and calculate the corresponding standard (error) uncertainties $S E\left(O_{r s}\right), S E\left(r_{s^{\prime} p}\right)$, and $S E\left(r_{s^{\prime \prime}} p^{\prime}\right)$ for each combination of $r \in\{u, v, w\}, s \neq s^{\prime} \neq s^{\prime \prime} \in\{x, y, z\}$, and $p, p^{\prime} \in\{1,2\}$.

If each system is solved independently, and if each of the sets $\left\{r_{s 1}, r_{s 2}, S E\left(r_{s 1}\right), S E\left(r_{s 2}\right)\right\}$ is consistent with the assumption that $r_{s 1}$ and $r_{s 2}$ are samples drawn from the same random distribution, 
for which $S E(r 1) \approx S E(r 2)$ is a necessary but not sufficient condition, then estimate the $s$ element of the $r$-accelerometer vector as

$$
r_{s}=\frac{r_{s 1}+r_{s 2}}{2}
$$

for $r \in\{u, v, w\}$ and $s \in\{x, y, z\}$, with an estimated standard (error) uncertainty of

$$
S E\left(r_{s}\right)=\sqrt{\frac{S E\left(r_{s 1}\right)^{2}+S E\left(r_{s 2}\right)^{2}}{2}} .
$$

If this assumption is not satisfied, then estimate $r_{S}$ and $S E\left(r_{S}\right)$ with suitable alternatives.

If all of the sets $\left\{O_{r x}, O_{r y}, O_{r z}, S E\left(O_{r x}\right), S E\left(O_{r y}\right)\right.$, and $\left.S E\left(O_{r z}\right)\right\}$ are consistent with the assumption that $O_{r x}, O_{r y}$, and $O_{r z}$ are samples drawn from the same random distribution, then estimate the $r$-offset signal as

$$
O_{r}=\frac{O_{r x}+O_{r y}+O_{r z}}{3}
$$

for $r \in\{u, v, w\}$, with an estimated standard (error) uncertainty of

$$
S E\left(O_{r}\right)=\sqrt{\frac{S E\left(O_{r x}\right)^{2}+S E\left(O_{r y}\right)^{2}+S E\left(O_{r z}\right)^{2}}{3}} .
$$

If this assumption is not satisfied, then estimate $O_{r}$ and $S E\left(O_{r}\right)$ with a suitable alternative that is consistent with the estimates of $O_{r}$ and $S E\left(O_{r}\right)$.

Intrinsic esponsivities The maximum responsivities of the three accelerometers, which are intrinsic parameters, are estimated separately as

$$
\begin{gathered}
u=\|\vec{u}\|=\sqrt{u_{x}^{2}+u_{y}^{2}+u_{z}^{2}} \\
v=\|\vec{v}\|=\sqrt{v_{x}^{2}+v_{y}^{2}+v_{z}^{2}} \\
w=\|\vec{w}\|=\sqrt{w_{x}^{2}+w_{y}^{2}+w_{z}^{2}},
\end{gathered}
$$

where the values of $u_{x}, \ldots, w_{z}$ are available directly from the solutions of Eq. (9) if the nine systems of linear equations represented by these equations were solved simultaneously, or from Eq. (10) if the nine systems of linear equations represented by Eq. (9) were solved independently. In either case, the estimated standard (error) uncertainty is given by

$$
S E(r)=\frac{\sqrt{\left[r_{x} S E\left(r_{x}\right)\right]^{2}+\left[r_{y} S E\left(r_{y}\right)\right]^{2}+\left[r_{z} S E\left(r_{z}\right)\right]^{2}}}{r} .
$$

Intrinsic angles The angles between the accelerometer axes can be calculated as

$$
\begin{aligned}
& \phi_{u v}=\arccos (\hat{u} \cdot \hat{v})=\arccos \left(\frac{\vec{u} \cdot \vec{v}}{\|u\|\|v\|}\right)=\arccos \left(\frac{u_{x} v_{x}+u_{y} v_{y}+u_{z} v_{z}}{u v}\right) \\
& \phi_{v w}=\arccos (\hat{v} \cdot \hat{w})=\arccos \left(\frac{\vec{v} \cdot \vec{w}}{\|v\|\|w\|}\right)=\arccos \left(\frac{v_{x} w_{x}+v_{y} w_{y}+v_{z} w_{z}}{v w}\right) \\
& \phi_{w u}=\arccos (\hat{w} \cdot \hat{u})=\arccos \left(\frac{\vec{w} \cdot \vec{u}}{\|w\|\|u\|}\right)=\arccos \left(\frac{w_{x} u_{x}+w_{y} u_{y}+w_{z} u_{z}}{w u}\right),
\end{aligned}
$$


where the values of $u, v$, and $w$ are available from Eq. (14), and, as already mentioned, the values of $u_{x}, \ldots, w_{z}$ are available directly from the solutions of Eq. (9) or Eq. (10). The standard (error) uncertainties of the intrinsic angles are given by

$$
S E\left(\phi_{r r^{\prime}}\right)=\frac{\sqrt{\left[\vec{r}_{s} \cdot S E\left(\vec{r}_{s}^{\prime}\right)\right]^{2}+\left[\vec{r}_{s}^{\prime} \cdot S E\left(\vec{r}_{s}\right)\right]^{2}+\cos \phi_{r r^{\prime}}\left[\left[r S E\left(r^{\prime}\right)\right]^{2}+\left[r^{\prime} S E(r)\right]^{2}\right]}}{r r^{\prime} \sin \phi_{r r^{\prime}}} .
$$

\section{Relation of the Intrinsic Parameters to the Conventional Cross-Sensitivity Matrix}

The conventional cross-sensitivity matrix $\mathscr{P}$ is used to calculate the acceleration input vector $\vec{a}$ that produces the measured offset-corrected output vector of the DUT given by $\vec{R}-\vec{O}$ with all quantities expressed in the $(x, y, z)$ gimbal coordinate system. Specifically,

$$
\left[\begin{array}{l}
a_{x} \\
a_{y} \\
a_{z}
\end{array}\right]=\left[\begin{array}{lll}
\rho_{x x} & \rho_{x y} & \rho_{x z} \\
\rho_{y x} & \rho_{y y} & \rho_{y z} \\
\rho_{z x} & \rho_{z y} & \rho_{z z}
\end{array}\right]\left[\begin{array}{l}
R_{x}-O_{x} \\
R_{y}-O_{y} \\
R_{z}-O_{z}
\end{array}\right]=\mathscr{P}\left[\begin{array}{l}
R_{x}-O_{x} \\
R_{y}-O_{y} \\
R_{z}-O_{z}
\end{array}\right] .
$$

On the other hand, the intrinsic responsivity vectors $\vec{u}, \vec{v}$, and $\vec{w}$ are used to calculate the predicted offset-corrected output from each DUT accelerometer. Specifically,

$$
\begin{aligned}
R_{u}-O_{u} & =\vec{u} \cdot \vec{a}=u_{x} a_{x}+u_{y} a_{y}+u_{z} a_{z} \\
R_{v}-O_{v} & =\vec{v} \cdot \vec{a}=v_{x} a_{x}+v_{y} a_{y}+v_{z} a_{z} \\
R_{w}-O_{w} & =\vec{w} \cdot \vec{a}=w_{x} a_{x}+w_{y} a_{Y}+w_{z} a_{z} .
\end{aligned}
$$

We define

$$
\mathscr{Q}=\left[\begin{array}{lll}
q_{x x} & q_{x y} & q_{x z} \\
q_{x y} & q_{y y} & q_{y z} \\
q_{x z} & q_{z y} & q_{z z}
\end{array}\right]=\mathscr{P}^{-1}
$$

to be the inverse of $\mathscr{P}$. Multiplication of Eq. (18) by $\mathscr{Q}$ followed by a rearrangement of terms gives

$$
\left[\begin{array}{l}
R_{x}-O_{x} \\
R_{y}-O_{y} \\
R_{z}-O_{z}
\end{array}\right]=\left[\begin{array}{ccc}
q_{x x} & q_{x y} & q_{x z} \\
q_{y x} & q_{y y} & q_{y z} \\
q_{z x} & q_{z y} & q_{z z}
\end{array}\right]\left[\begin{array}{l}
a_{x} \\
a_{y} \\
a_{z}
\end{array}\right]=\left[\begin{array}{c}
q_{x x} a_{x}+q_{x y} a_{y}+q_{x z} a_{z} \\
q_{y x} a_{x}+q_{y y} a_{y}+q_{y z} a_{z} \\
q_{z x} a_{x}+q_{z y} a_{y}+q_{z z} a_{z}
\end{array}\right]
$$

Equations (18) and (21) have the same form but complementary interpretations. The latter can be read as "response = responsivity multiplied by stimulus (acceleration in this case)," and the former can be read as "stimulus equals sensitivity multiplied by response." Comparison of the right-hand sides of Eq. (21) and Eq. (19) shows that

$$
\left[\begin{array}{ccc}
\rho_{x x} & \rho_{x y} & \rho_{x z} \\
\rho_{y x} & \rho_{y y} & \rho_{y z} \\
\rho_{z x} & \rho_{z y} & \rho_{z z}
\end{array}\right]^{-1}=\mathscr{P}^{-1}=\mathscr{Q}=\left[\begin{array}{ccc}
q_{x x} & q_{x y} & q_{x z} \\
q_{y x} & q_{y y} & q_{y z} \\
q_{z x} & q_{z y} & q_{z z}
\end{array}\right]=\left[\begin{array}{ccc}
u_{x} & u_{y} & u_{z} \\
v_{x} & v_{y} & v_{z} \\
w_{x} & w_{y} & w_{z}
\end{array}\right],
$$

which establishes the relation of the elements of the cross-sensitivity matrix to the elements of the intrinsic responsivity vectors expressed in the same coordinate system. Equation (22) and Eqs. (9-17) are the top-level results of this report.

*Equation 21 corrected on March 6, 2019, by adding plus signs to the right matrix 


\section{Relation to Previous Work}

None of our references explicitly mentions the intrinsic parameters or their potential uses. Of the references cited here, our work is most like that of Ref. [2]. However, that work is optimized for efficiency in terms of time and computational complexity, requiring only manual rotations of the DUT around each of three nominally orthogonal axes while lying on a flat surface. By contrast, our work is optimized for minimizing the uncertainties in determinations of the intrinsic parameters of three-axis accelerometers and relating the intrinsic parameters to the elements of the cross-sensitivity matrix.

Another important difference is that [2] mentions some ideas related to the intrinsic parameters in passing, whereas we focus directly on the nine intrinsic parameters. For instance, [2] distinguishes between sensor-sensitivity axes and sensor-coordinate axes and calculates angles between the former and the latter, but it does not calculate the angles between the sensor-sensitivity axes, which are the intrinsic angles.

IEEE standard 1293 [1] provides a standard nomenclature, a format for reporting, and informative examples of measurement and analysis procedures for linear single-axis, nongyroscopic accelerometers. The focus of that work is generality, covering a wide range of accelerometer types and sources of error. Despite the apparent limitation to "linear single-axis accelerometers" implied by the title, nonlinearity errors and two- and three-axis devices are covered extensively in the annexes. The work described in our manuscript as well as that in the references given here and that in the references cited in those references typically describe optimizations for specific applications, extensions, or replacements of the material in standard 1293.

References [4] and [3] treat high-accuracy inertial navigation systems. The former is optimized for self-calibrating rotating systems composed of three nominally orthogonal accelerometers and three nominally orthogonal gyroscopes. The latter is optimized for systems composed of three nominally orthogonal, spatially separated accelerometers without gyroscopes. Reference [5] describes electronic procedures for calibrating capacitive accelerometers without any motion. Reference [6] describes an alternative procedure for aligning the gimbal axes with the local gravitational field instead of that given in Appendix B.

\section{Discussion and Conclusion}

Equations (5-17) parameterize the response of a three-axis accelerometer DUT in terms of the intrinsic (not necessarily orthogonal) coordinate system of the DUT plus the intrinsic background (DC offset) signals $O_{u}, O_{v}$, and $O_{w}$ from the three accelerometers. The goal of this parameterization is the set of nine intrinsic parameters listed in Table 2.

Table 2. The three intrinsic offset parameters of a three-axis accelerometer are the offset signals of the individual accelerometers. The three intrinsic responsivity parameters are the responsivities of the individual accelerometers along their axes of maximum responsivity, and the three intrinsic angles are the angles between the axes of maximum responsivity.

\begin{tabular}{|c|c|c|}
\hline \multicolumn{3}{|c|}{ Intrinsic Parameters } \\
\hline $\begin{array}{c}\text { Intrinsic } \\
\text { Offsets }\end{array}$ & $\begin{array}{c}\text { Intrinsic } \\
\text { Responsivities }\end{array}$ & $\begin{array}{c}\text { Intrinsic } \\
\text { Angles }\end{array}$ \\
\hline \hline$O_{u}$ & $u$ & $\phi_{u v}$ \\
$O_{v}$ & $v$ & $\phi_{v w}$ \\
$O_{w}$ & $w$ & $\phi_{u w}$ \\
\hline
\end{tabular}




\section{Journal of Research of National Institute of Standards and Technology}

The intrinsic responsivities can be measured directly or calculated from a conventional cross-sensitivity matrix $\mathscr{P}$ as

$$
\left[\begin{array}{ccc}
u_{x} & u_{y} & u_{z} \\
v_{x} & v_{y} & v_{z} \\
w_{x} & w_{y} & w_{z}
\end{array}\right]=\mathscr{P}^{-1}
$$

and the cross-sensitivity matrix can be calculated from the intrinsic responsivities by inverting both sides of Eq. (23).

The measurement geometry described in Eq. (5) is optimized for calculating the intrinsic parameters of a DUT. However, since this parameterization is independent of the DUT coordinates in any given coordinate system, it is not expected to be of use in applications of three-axis accelerometers. Instead, it is expected to be useful in comparisons of cross-sensitivity measurement capability.

For instance, suppose measurements by two different laboratories of the cross-sensitivity matrix of the same three-axis accelerometer agree very well for the on-axis elements but agree much less well for the off-axis elements. Comparison of the intrinsic parameters of the DUT that can be calculated from the two different cross-sensitivity matrices will isolate the differences caused by gimbal-associated errors from those caused by differences in the DUT-gimbal alignment present during the different measurements.

Therefore, an interlaboratory comparison of cross-sensitivity could be organized by a central laboratory that would carry out a very accurate determination of the intrinsic parameters of one (or more) DUTs. It would then distribute that DUT to participating laboratories that would measure the cross-sensitivity following their standard procedures. Comparison of the intrinsic parameters calculated from the cross-responsivity matrices reported by the different laboratories would establish the state of the gimbal-related measurement errors, and the remaining differences in the cross-sensitivity results could be reliably assigned to alignment differences.

Another potential use is to distinguish packaging-induced strain from misalignment during packaging of three-axis accelerometers for which the cross-sensitivity matrix has been measured in a precisely aligned batch process prior to packaging. Suppose a manufacturer has developed a batch process to simultaneously measure the cross-sensitivity of a large number of three-axis accelerometers in such a way that the alignment relative to the measuring instrument can be repeated during packaging. Further suppose that the cross-sensitivity matrix of a small sample of packaged devices is measured, and the results differ from the batch-process measurement results by more than some specified tolerances.

Comparison of the intrinsic parameters calculated from the cross-sensitivities measured before and after packaging can provide a diagnostic on the packaging process. If the difference between all of the before-and-after intrinsic parameters falls within some specified tolerances, then the packaging process is not reproducing the alignment in the batch measurement as well as desired. On the other hand, if the differences between some of the before-and-after intrinsic parameters fall outside the specified tolerances, then the packaging process is straining the accelerometer die more than expected. In either case, the manufacturer will know what aspect of the packaging process needs to be improved. 


\section{Appendix A. Aligning the Gimbal Axes with Gravity}

This appendix describes a simple method to align the three gimbal axes with gravity as shown in Fig. $2 \mathbf{A}, 2 \mathbf{B}$, and $2 \mathbf{C}$ subject to two assumptions. The first is that the three gimbal axes are orthogonal with uncertainties well less than those desired in the uncertainties of the angles between any two accelerometer axes. The second is that the uncertainty of a $90^{\circ}$ rotation around each axis capable of $360^{\circ}$ is also well less than that desired in the uncertainty in the angles between any two accelerometer axes. Ideally, it should be necessary to align the gimbal $z$-axis with the local gravitational field only once, but it would be prudent to check the alignment periodically.

Let $R_{u}\left(\alpha_{x}^{\prime}, \alpha_{y}^{\prime}, \beta_{z}^{\prime}\right)$ and $R_{v}\left(\alpha_{x}^{\prime}, \alpha_{y}^{\prime}, \beta_{z}^{\prime}\right)$ be the respective responses of the $u$-axis and $v$-axis accelerometers as a function of angles $\alpha_{x}^{\prime}, \alpha_{y}^{\prime}$, and $\beta_{z}^{\prime}$ when the gimbal axes are in orientation $\mathbf{A}$ defined in Fig. 2 starting from configuration 1 of Fig. 1. In this case,

$$
\begin{gathered}
\gamma_{x}^{\prime} \approx \arcsin \left(\frac{R_{v}\left(0^{\circ}, 0^{\circ}, 0^{\circ}\right)-R_{v}\left(0^{\circ}, 0^{\circ}, 180^{\circ}\right)}{R_{v}\left(270^{\circ}, 0^{\circ}, 0^{\circ}\right)-R_{v}\left(90^{\circ}, 0^{\circ}, 0^{\circ}\right)}\right) \\
\gamma_{y}^{\prime} \approx \arcsin \left(\frac{R_{u}\left(0^{\circ}, 0^{\circ}, 0^{\circ}\right)-R_{u}\left(0^{\circ}, 0^{\circ}, 180^{\circ}\right)}{R_{u}\left(0^{\circ}, 270^{\circ}, 270^{\circ}\right)-R_{u}\left(0^{\circ}, 90^{\circ}, 270^{\circ}\right)}\right)
\end{gathered}
$$

which approximate the angles through which the gimbal must be rotated around axes parallel to the gimbal $x$ and $y$ axes, respectively, to align the gimbal $z$ axis with the local gravitational field. Since these expressions are not exact, nominal values determined from a small number of replications can be used for the quantities in the denominators, and a few rapidly converging iterations may be required to obtain to the minimum-error alignment.

Depending upon the nature of the gimbal being used, the required tilting can be implemented with leveling axes provided specifically for this purpose. If no leveling axes are available, then one of the required tilts can be obtained by redefining $\alpha_{x}^{\prime}$ such that $\gamma_{x}^{\prime}=0$. Due to constraints imposed by a two-axis gimbal, the required rotation through $\gamma_{y}^{\prime}$ cannot be implemented by a rotation of $\alpha_{y}^{\prime}$. The only solution would be to place shims under one of the edges of the gimbal that is parallel to the $y$ axis when the gimbal is in orientation $\mathbf{A}$ of configuration 1. This is not only much less convenient, but also more difficult to execute with high precision. This is the reason that many gimbals provide a leveling axis with a limited range but sufficient precision to complete the alignment of the gimbal $z$ axis with the local gravitational field.

Assume that the procedure described above has been completed. In this case, the standard error uncertainties in the $x$ - and $y$-axis angles between the $z$ axis of the gimbal and the gravity unit vector are given by the standard-error uncertainties $S E\left(\gamma_{y}^{\prime}\right)$ and $S E\left(\gamma_{x}^{\prime}\right)$, respectively. If both the uncertainty in the orthogonality of the gimbal axes and the uncertainty in the gimbal rotation-angle settings are small compared to the uncertainty in the alignment of the $z$ axis with the gravity unit vector $\hat{g}$, then the uncertainty of the alignment of the $y$ axis of the gimbal with $\hat{g}$ will be comparable to that obtained in the alignment of the $z$ axis of the gimbal with $\hat{g}$.

\section{Appendix B. Interpretation of the Residuals of the Fits}

As mentioned at the end of Sec. 2, the measurement and analysis procedures described here provide checks on and measures of the cumulative effect of all of the errors that are not included in the models of the DUT that are fit to the measured data. This discussion will be presented in terms of measurements of the response of the $w$ accelerometer to rotation about the $x$ axis, but the results can be readily generalized to measurements of the response of the $r$ accelerometer to rotation about the $s$ axis.

The residuals of the individual measurements of $R_{w m}\left(\alpha_{x n}\right)$ for $m=1, \cdots, M$ should be tested for no correlation and normality before being averaged. If they fail the test for no correlation, then the 
sampling rate or some similar parameter of the data acquisition system should be modified accordingly. If they pass the test for no correlation, then the test for normality can identify other problems that need to be addressed such as occasional outliers or nonnegligible offset drift during the measurement period. Once all of the $R_{w m}\left(\alpha_{x n}\right)$ data passes the tests for no correlation and normality, the model equation can be fit to the data. If the variance of the residuals of the fit is significantly larger than the quadrature sum of the variances of the residuals of the $M$ measurements of $R_{w m}\left(\alpha_{x n}\right)$ at each $\alpha_{x n}$, then the residuals of the fit contain information about deviations of the accelerometer response from the simple linear model of Eq. (9).

These deviations can be divided into two classes. The first class includes deviations of the performance of the gimbal from the assumed ideal, that are implicit in Eq. (5). These include nonorthogonality of the gimbal axes, errors in setting the gimbal angles, and errors in aligning the gimbal axes with the gravitational field. The second class includes deviations of the performance of the accelerometers from the assumed ideal, that are implicit in Eq. (9). These include but are not limited to nonunique directions of maximum responsivity of the gimbal accelerometers, offset drifts large enough relative to noise to alter the assumed normal distribution of the values of $R_{w}$, but small enough to remain hidden in the $R_{w m}\left(\alpha_{x n}\right)$ data at each angle, and various types of nonlinearity. Small nonlinearities show up as a sinewave in the residuals. Clipping causes clumps of data at the extremes of the residuals and dead-band causes clumping of data around some point (often zero) away from the extremes of the residuals.

\section{References}

[1] Institute of Electrical and Electronics Engineers (1998) Standard IEEE 1293-1998-IEEE Standard Specification Format Guide and Test Procedure for Linear, Single-Axis, Non-Gyroscopic Accelerometers (Institute of Electrical and Electronics Engineers, New York, New York).

[2] Stančin S, Tomažič S (2014) Time- and Computation-Efficient Calibration of MEMS 3D Accelerometers and Gyroscopes. Sensors 14:14885-14915, https://doi.org/10.3390/s140814885, and references therein.

[3] Schopp P, Graf H, Burgard W, Manoli Y (2016) Self-Calibration of Accelerometer Arrays. IEEE Transactions on Instruments and Measurement 65(8):1913-1925, https://doi.org/10.1109/TIM.2016.2549758, and references therein.

[4] Gao P, Li K, Wang L, Liu Z (2017) A Self-Calibration Method for Accelerometer Nonlinearity Errors in Triaxial Rotational Inertial Navigation System. IEEE Transactions on Instruments and Measurement 66(2):243-252, https://doi.org/10.1109/TIM.2016.2625958, and references therein.

[5] Dar T, Suryanarayanan K, Geisberger A (2014) No Physical Stimulus Testing and Calibration for MEMS Accelerometer. Journal of Microelectromechanical Systems 23(4):811-818, https://doi.org/10.1109/JMEMS.2013.2294562, and references therein.

[6] Fang J, Liu Z (2015) A New Inclination Error Calibration Method of Motions Table Based on Accelerometers. IEEE Transactions on Instruments and Measurement 64(2):487-493, https://doi.org/10.1109/TIM.2014.2347219.

About the authors: Jon Geist is an electrical engineer in the Nanoscale Metrology Group in the Engineering Physics Division at NIST. Dr. Geist's current activities include the development of improved acceleration measurement methods and the use of localization microscopy to measure microand nano-scale motion in microsystems.

M. Yaqub Afridi is a NIST Associate working in the Acceleration, Vibration, and Acoustics Project under a grant to Theiss Research from the Nanoscale Metrology Group. Dr. Afridi's areas of expertise are analog and digital interface circuit design and fabrication technologies for microsensor. His current research efforts are directed at improving the precision, accuracy, and usability of inertial, vibration, and shock measurements and calibrations.

Craig McGray, who is Principal Engineer at Modern Microsystems, Inc. , is also a NIST Associate working in the Nanoscale Metrology Group. Dr. McGray has worked on a number of different projects in the Engineering Physics Division, including inertial measurements and localization microscopy.

Michael Gaitan leads the NIST Physical Measurements Laboratory's Acceleration, Vibration, and Acoustics Project. Dr. Gaitan is active in the development of new microsensor technologies as well as 
Volume 122, Article No. 32 (2017) https://doi.org/10.6028/jres.122.032

Journal of Research of National Institute of Standards and Technology

calibration and test methods that are required for ensuring high accuracy measurements that enhance economic security and improve our quality of life.

The National Institute of Standards and Technology is an agency of the U.S. Department of Commerce. 